Gerät die Vorteile der maßanalytischen Bestimmung zunutze, wobei sich die Verwendung einer Mikrowaage erübrigt und auch Zeit gespart wird. Das Gerät besteht aus zwei zylindrisch geformten Elektrodengefäßen mit Ablaßhähnen, die durch ein horizontales Rohr mit Einfülltrichter miteinander verbunden sind. Die Flüssigkeiten in den Elektrodengefäßen sind durch zwei eingeschmolzene SinterglasDiaphragmen getrennt. Die Elelktroden aus Platinblech befinden sich im unteren Teil der Elektrodengefäße. Zur Messung füllt man das ganze Gerät mit Vanadium(IV)-sulfatlösung. Bei Stromdurchgang wird eine entsprechende Menge Vanadium an der Anode von der vierwertigen zur fünfwertigen Stufe oxydiert. Anschließend titriert man die Anodenflüssigkeit mit Eisen(II)-sulfatlösung und errechnet aus dem verbrauchten Volumen der Maßlösung die Strommenge. Die Bedingungen, unter denen die anodische Oxydation des Vanadyl-Ions mit 100\%iger Stromausbeute verläuft, wurden in einer Reihe von Versuchen ermittelt, bei denen das neue Coulometer mit einem Silbercoulometer im gleichen Stromkreis hintereinandergeschaltet war. Nach den Ergebnissen darf in einer an vierwertigem Vanadium 0,02 normalen Lösung die Konzentration an Schwefelsäure nicht höher als 0,3 molar und die Stromdichte nicht größer als $0,3 \mathrm{~mA} / \mathrm{cm}^{2}$ sein.

Die Vanadium(IV)-Lösung wird aus einer Ammoniumvanadatlösung durch Zusatz der berechneten Menge Schwefelsäure und Reduktion mit Schwefeldioxyd hergestellt. Die Vollständigkeit der Reduktion prüft man, indem man einen Tropfen der Lösung mit einem. Tropfen $0,2 \%$ iger Phenylanthranilsäurelösung antüpfelt, wobei keine Rotfärbung mehr auftreten darf. Der Überschuß an Schwefeldioxyd wird anschließend unter Durchleiten von reinem Kohlendioxyd verkocht. Die Eisen(II)-sulfatlösung wird aus MoHrschem Salz hergestellt und soll an Schwefelsäure 6 fach normal sein. Zur Anodenflüssigkeit wird vor der Titration soviel Schwefelsäure zugesetzt, daß sie einschließlich Waschwasser an Säure 10-12 fach normal ist.

Die gleichzeitig an der Kathode stattfindende Reduktion zur Vanadium(III)stufe verläuft, offenbar infolge von Nebenreaktionen, nicht mit 100\%iger Stromausbeute. Analoge Versuche mit einer Lösung von Cer(IV)-salz als Elektrolyt ergaben stark streuende Werte. Die Störung ist vielleicht darauf zurückzuführen, daß die Lösung außer Cer noch größere Mengen anderer seltener Erden enthielt.

ANTONI.

Von einer neuen Substanz als Ionenreduktor berichtet BARBRo HwLGER ${ }^{1}$. Während Cadmium ein zähes Metall ist, welches sich schwer fein verteilen läßt und bei der Aufladung blank wird, wodurch das Reduktionsvermögen verschlechtert wird, ist Cadmiumamalgan mit $20-22 \%$ Cd eine spröde Substanz, die sich noch leichter fein verteilen läßt, als das bisher schon zur Reduktion von Eisen verwendete flüssige Cadmiumamalgan mit etwa $3 \%$ Cd. Das Cd-reiche Amalgam läßt sich im Laboratorium leicht herstellen. Einige Stücke granulierten Cadmiums werden in einem kleinen bedeckten Becherglas mit $5 \%$ iger $\mathrm{H}_{2} \mathrm{SO}_{4}$ und $1 / 2 \mathrm{ml}$ Quecksilber versetzt und $5 \mathrm{~min}$ anf $100^{\circ} \mathrm{C}$ erwärmt. Hierbei lösen $4 \mathrm{~g} \mathrm{Hg}$ etwa $1 \mathrm{~g} \mathrm{Cd}$. Das überschüssige Cd bleibt als feste Substanz zurück. Einige Tropfen des flüssigen Amalgams werden etwa $1 \mathrm{~mm}$ dick in einen Mörser gegossen, solange das Material noch warm und formbar ist. Es kann dann leicht zu der gewünschten Korngröße aufgerieben werden. Die Säure wird in das Becherglas zurückgegeben und wenn noch überschüssiges $\mathrm{Cd}$ vorhanden ist, setzt man noch höchstens $1 / 4 \mathrm{ml} \mathrm{Hg}$ hinzu und wiederholt den Vorgang. Ein Reduktor von $2 \mathrm{ml}$ Inhalt in einer Höhe von $20 \mathrm{~cm}$ mit Cadmiumamalgam von $1 / 2 \mathrm{~mm}$ Korngröße gefüllt bewirkt die vollständige Reduktion einer Lösung von $5 \mathrm{mg} \mathrm{Fe} / \mathrm{ml}$ bei einer Durchlaufgeschwindigkeit von 3 Tropfen/sec

1 Svensk. kem. Tidskr. 61, 62 (1949). 
2-10 Vol\%iger Sohwefel- oder Salzsäure bei Zimmertemperatur. Sechswertiges: Uran wird unter denselben Bedingungen zu Uran(IV), zum geringen Teil auch zu: Uran(III) reduziert. Die Reduktionen verlaufen ohne Gasentwicklung.

\section{B. Frestentus.}

Für die Kalibrierung von Mikrobüretten und Mikroinjektionspipetten im Bereich: von $0,1-10 \mu l$ geben J. B. Buck, M. L. KeIstrer und M. R. ZkLle ${ }^{1}$ ein einfaches: Verfahren an. Es beruht auf der Tatsache, daß die von sehr kleinen Tropfen einer Farblösung auf Filtrierpapier hinterlassenen Flecke bei gleichbleibender Temperatur und Feuchtigkeit den entsprechenden. Volumina exponentiell proportional sind. Man kann auf diese Art Volumänderungen bis zu $0,1 \mu$ verfolgen. A. KURTENACkER.

Ober die Methode, Radiokohlenstoff ${ }^{14} \mathrm{C}$ quantitativ im GaszähIrohr zu messen, berichtet eingehend M. L. ErDINOFF ${ }^{2}$. Diese Art der Messung besitzt den Vorzug, da f die Menge des in das Zählrohr eingebrachten ${ }^{14} \mathrm{CO}_{2}$ leicht durch den Gasdruck: abzulesen ist und damit entgegen der üblichen radiometrischen Bestimmung als $\mathrm{Ba}^{14} \mathrm{CO}_{3}$-Niedersehlag Arbeitsaufwand und Zeit gespart wird. Das Zählrohr ist ein: übliches Gaszählrohr mit $\mathrm{CS}_{2}-\mathrm{CO}_{2}$-Füllung.

Der technische Aufbau der gesamten Apparatur wird eingehend beschrieben.

$\mathrm{Ba}^{14} \mathrm{CO}_{3}$ wird im geschlossenen. System nit $\mathrm{HClO}_{4}$ zersetzt und das entstandene ${ }^{14} \mathrm{CO}_{2}$ im gereinigten $\mathrm{N}_{2}$-Strom über mehrere Fallen und Mischgefäße in das Zäh/rohr. eingebracht. Die spezifische Aktivität läßt sich durch Beimischen von inaktivem: $\mathrm{CO}_{2}$ in weiten Grenzen regeln. Die Messungen wurden mit einem modifizierten Zählgerät (NEHER-HAPER-Schaltung) vorgenommen. Die Zählrohreinsatzspannungenliegen sehr hoch und verlangen bei höheren Gasdrucken ein spezielles Hochspannungsgerät von Spannungen bis über 6000 Volt.

Die Meßergebnisse bestätigen direkte Proportionalitäten zwischen der Teilchenzahl und eingebrachter ${ }^{14} \mathrm{CO}_{2}$-Menge in dem groBen Druckintervall von $2-175 \mathrm{~cm}$ $\mathrm{Hg}$. Bei geringen Gasdrueken liegt die mittlere Abweichung der Messungen vom: Sollwerte um 1,2\%. Dieser Fehler wird bei einem Gasdruck um $50 \mathrm{~cm} \mathrm{Hg-Säule-}$ noch weiter bis auf $0,4 \%$ herabgedrückt.

An Hand zahlreicher Zählrohrcharakteristiken und Eichkurven zeigt der Verfasser die Leistungsfähigkeit dieser Meßmethode. Die einzelnen Meßpunkte enthalten durchschnittlich 30000 Teilchen (gezählt), der statistische Fehler wird berechnet.

Festgestellt wurde ferner, daß die Apparatur nach 5 min langem Ausspülen mit. inaktivem $\mathrm{CO}_{2}-\mathrm{N}_{2}$.Gemisch keine Restaktivität mehr zeigte und die an der Wandung. adsorbierte ${ }^{14} \mathrm{CO}_{2}$-Aktivität in jedem Fall vernachlässigbar klein wird.

AbschlieBend weist der Verfasser darauf hin, daß mit seiner Anordnung (ein 28 ml-Zählrohr mit 2 Atmosphären $\mathrm{CO}_{2}$-Druck) noch eine spezifische Aktivität von. $4 \times 10^{-10}$ Curie per Gramm C mit etwa $10 \%$ Fehler zu zählen gestattet, während die Grenze der ${ }^{14} \mathrm{C}$-Bestimmung mit dem $2 \mathrm{mg} / \mathrm{cm}^{2}{ }^{2}$ Glockenzählrohr bei etwa $7,4 \times 10^{-8}$ Curie/g Kohlenstoff liegt.

W. HeRr.

Um die Bestimmung von Gefrier- und Schmelzpunkten unter der Eigenatmosphäre der Analysensubstanz, d. $h$. in Abwesenheit von Luft oder anderen Fremdgasen durchführen zu können, gestalten A. R. GLasGow, jr., N. C. KrouskoPund F. D. Rossrst ${ }^{3}$ ihren früher ${ }^{4}$ beschriebenen Apparat dahingehend um, das im Vakuum gearbeitet werden kann. Ein Dewargefäß von $30 \mathrm{~mm}$ lichter Weite des:

1 Analytica Chimica Acta 4, 130 (1950).

2 Analytic. Chemistry 22, 529 (1950).

3 Analytic. Chemistry 22, 1521 (1950).

4 Analytic. Chemistry 20, 410 (1948). 